American Journal of Pharmacology and Toxicology 3 (4): 225-228, 2008

ISSN 1557-4962

(C) 2008 Science Publications

\title{
Extraction of Essential Oil from Nigella sativa Using Supercritical Carbon Dioxide: Study of Antibacterial Activity
}

\author{
${ }^{1}$ Nagi A. Alhaj, ${ }^{2}$ Mariana N. Shamsudin, ${ }^{2}$ Hana F. Zamri and ${ }^{3}$ Rasedee Abdullah \\ ${ }^{1}$ Laboratory Immunotherapeutic and Vaccine, Institute of Bioscience \\ ${ }^{2}$ Department of Microbiology and Parasitology, Faculty of Medicine and Heath Sciences \\ ${ }^{3}$ Department of Microbiology and Pathology, Faculty of Veterinary Medicine, \\ University Putra Malaysia 43400 Serdang, Selangor DE, Malaysia
}

\begin{abstract}
The antimicrobial activity of $N$. sativa essential oil obtained by supercritical fluid extraction by carbon dioxide was investigated against Gram Positive and Gram negative strains, isolated from clinical specimens. Best conditions for Black cumin oil extraction are obtained at 400 bar, $40^{\circ} \mathrm{C}$ and a solvent flow rate of $25 \mathrm{~g} \mathrm{~min}^{-1}$. The seed extracts were prepared by supercritical fluid extraction method. Filter paper discs impregnated with varying concentrations of $N$. sativa extract were tested by the disk diffusion method. Methicillin Resistant Staphylococcus Aureus (MRSA) ATCC strain (700968), E. coli ATCC strain (25922), E. coli 0157 ATCC strain (12799), Extended-Spectrum BetaLactamase (ESBL) Klebsiella pneumoniae ATCC strain (700603), Carbapenam Resistant acenitobacter Baumanii (CRAB) clinical strain and Vibrio cholerae 01 Ogawa and 0139 Bengal clinical strains were investigated. The inhibition zones of the Mueller Hinton agar in different extract concentrate ion showed that at $25 \mathrm{mg} 20 \mu \mathrm{L}^{-1}, 50 \mathrm{mg} 20 \mu \mathrm{L}^{-1}$ and $100 \mathrm{mg} 20 \mu \mathrm{L}^{-1}$, the inhibition zones increased accordingly in $S$. aureu. However, $N$. sativa was found to be inactive against ESBL producers (E. coli and K. pneumoniae).
\end{abstract}

Key word: Supercritical fluid extraction, nigella sativa, antimicrobial activity, gram positive and gram negative bacteria, university putra Malaysia, Yemen

\section{INTRODUCTION}

The extraction of essential oil components using solvent at high pressure, or Super-Critical Fluids (SCF), has received much attention in the past several years, especially in food, pharmaceutical and cosmetic industries, because it presents an alternative to conventional processes such as organic solvent extraction and steam distillation ${ }^{[5-8]}$. Supercritical fluid extraction allows a continuous modification of solvent power and selectivity by changing the solvent density ${ }^{[4,12,14]}$. Black cumin is an economically important umbel lifer growing wild in the dry temperature regions of Jammu-Kashmir, Himachal Pradesh, Afghanistan, Baluchestan and Iran. The seeds, rich in essential oil, are consumed widely as condiment. In the indigenous system of medicines, seeds are regarded as stimulants and carminatives and found to be useful in diarrhoea and dyspepsia ${ }^{[1]}$. Also, this plant is used for culinary purposes and for flavouring foods and beverages. It should be noted that the name of black cumin is sometimes given to entirely unrelated spice
Nigella sativa. The crude extracts of N. Sativa were reported to have a promising effect on multi-drug resistant S. aureus and Candida albicans ${ }^{[10]}$, Shigella spp., Vibrio cholerae ${ }^{[15]}$. Production of $\beta$ lactamases is the most common mechanism of resistance among the Gram-negative. The vast majority of strains expressing these enzymes belong to the family Enterobacteriacea like $K$. pneumonia and some E. coli strains, $\beta$-lactam antibiotics are the most common treatment for bacterial infections. Extended-Spectrum $\beta$ Lactamases (ESBL) have become a widespread serious problem and several aspects of them are worrying. The potential use of alternative antibiotics in drug-resistant bacteria from various plant extracts have been studied by many researches. To document the antibacterial effects of $N$. sativa both gram positive and gram negative bacteria were tested.

\section{MATERIALS AND METHODS}

Nigella sativa seeds: The seeds were purchased from a local herbal shop in Yemen. Seeds were stored in dark

Corresponding Author: Rasedee Abdullah, Department of Microbiology and Pathology. Faculty of Veterinary Medicine, University Putra Malaysia 43400 Serdang, Selangor DE, Malaysia Tel: 603-89441582 
at $4{ }^{\circ} \mathrm{C}$ for 20 days. Immediately prior to the extraction process, the lseeds were ground in a blender to produce a powder with an approximate size of $150 \mathrm{~g}$.

Supercritical Fluid Extraction (SFE): A Suprex MPS/225 system (Thar, Germany) at the Food science and biotechnology Laboratory UPM in the SFE mode was used for the extraction. In this study, extraction was performed by filling extraction vessel with $150.0 \mathrm{~g}$ of the completely crashed seeds. The plant was then extracted with SFE under 400 atm pressure and $40^{\circ} \mathrm{C}$ temperature for $15 \mathrm{~min}$ static followed by $20 \mathrm{~min}$ dynamic. A Duraflow manual variable restrictor (Suprex) was used in the SFE system to collect the extracted analytes. The SFE flow rate through the Duraflow restrictor was approximately $25 \mathrm{~g} \mathrm{~min} \mathrm{~min}^{-1}$ (compressed). The extracted analytes were collected in a volumetric flask. The final volume of the extract was adjusted to $35 \mathrm{~mL}$ at the end of the extraction. In order to have better collection efficiency, the $35 \mathrm{~mL}$ volumetric flask was placed in an ice bath during the dynamic extraction stage.

Antimicrobial Susceptibility Testing (AST) by NCCLS and concentrations of extracts: Extracts were diluted two-fold and used at a concentration starting from $100 \%$ to an end dilution of $6.25 \%$ where $25 \mathrm{mg} \mathrm{mL}^{-1}$ in $10 \%$ Dimethyl Sulfoxside (DMSO) were prepared using vortex mixture. Whatman AA paper discs were injected with $20 \mu \mathrm{L}$ of different $N$. sativa extracts using a micropipette and were dried in the biosafety cabinet for $30 \mathrm{~min}$. Negative control disc were prepared using a 10\% DMSO. All the bacteria were cultured on Nutrient Agar (NA) (Merck). Inoculums were prepared in $5 \mathrm{~mL}$ Mueller Hinlon broth with 3-5 colonies of each bacterial strain. The inoculums were incubated at $35^{\circ} \mathrm{C}$ for $2-3 \mathrm{~h}$ to get an approximately close to $0.5 \mathrm{McFarland}$ standard for susceptibility testing ${ }^{[13]}$. Two methods were used- 1) Disc diffusion method: Sterile paper discs impregnated with each extract were placed aseptically over the bacterial cultures and incubated at $37^{\circ} \mathrm{C}$ for 24 h. 2) Well diffusion method: wells were made on inoculated agar and each well filled with $25 \mu \mathrm{L}$ of extract. Each inoculated plate was incubated with a positive-control antibiotic disc for each of the bacteria.

Bacterial strains: The bacterial strain were used Methicillin Resistant Staphylococcus Aureus (MRSA) ATCC strain 700968, E. coli ATCC strain 25922, E. coli 0157 ATCC strain 12799, Extended-Spectrum Beta-Lactamase (ESBL) Klebsiella pneumoniae ATCC strain 700603, carbapenam Resistant Acenitobacter Baumanii (CRAB) clinical strain and
Vibrio cholerae 01 Ogawa and 0139 Bengal clinical strains. Purchased from (Biosyntech Malaysia) and kept at the Medical Microbiology Laboratory, University Putra Malaysia (UPM).

\section{RESULTS}

All the bacteria tested as shown in the Table 1, only Methicillin Resistant Staphylococcus Aureus (MRSA) showed high susceptibility towards the extract the inhibition zones increased accordingly in $S$. aureu. However, $N$. sativa was found to be inactive against ESBL producers (E. coli and K. pneumoniae). For the disc diffusion method, MRSA showed large zones of inhibition for the first three dilutions (100, 50 and 25\% concentration) and showed resistance for the last two dilutions. For the well diffusion method, all wells produced large zones of inhibition and the inoculated plate itself produced little or almost no growth of MRSA (Fig. 1 a-d). V. cholerae plates were tested using

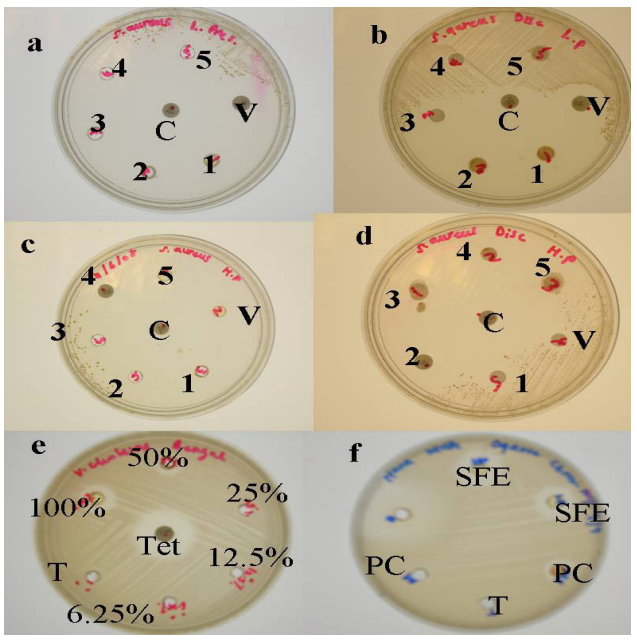

Fig. 1: Susceptibility of MRSA towards extractsConcentrations 1- 100, 2- 50, 3- 25, 4- 12.5 and 5- 6.25\%; V- Vancomycin, C- Cefoxitin: (a): Well diffusion of SCF extract; (b): Disc diffusion of oil extract; (c): Well diffusion of SFE extract; (d): Disc diffusion of SFE extract. (e and f): Susceptibility of $V$. cholerae towards extracts: (e): Well diffusion with different concentrations of extract on V.cholerae 0139 (Bengal)-100, 50, 25, 12.5 and $6.25 \%$ concentration, Tettetracycline, T- Tryptic soy broth (as solvent negative control); Inhibition zone diameter at $100 \%$ extract concentration is $13 \mathrm{mM}$; at $50 \%$ concentration is $9 \mathrm{mM}$ (f): Well diffusion of $100 \%$ (PC pure control of DMSO) extracts on $V$. cholerae 01 (Ogawa), T- Tryptic soy broth 
Am. J. Pharm. \& Toxicol., 3 (4): 225-228, 2008

Table 1: Commercial antibiotic discs were included as controls

\begin{tabular}{lll}
\hline Bacteria & Antibiotic control & SFE extract \\
\hline MRSA & Cefoxitin/ & Sensitive \\
Eancomycin & \\
E. coli 25922 & Gentamycin & Resistant \\
E. coli 12799 H7 & Gentamycin & Resistant \\
ESBL K. pneumoniae & Gentamycin & Resistant \\
& $\begin{array}{l}\text { Cefotaxime/ } \\
\text { Ceftazidime }\end{array}$ & Resistant \\
CRAB & Imipenam/ & Resistant \\
& Carbapenam & \\
V. cholerae Bengal & Tetracycline & Small inhibition zone \\
\hline
\end{tabular}

only the well diffusion method, where $V$. cholerae 0139 Bengal was tested with different extract concentrations (100, 50, 25, 12.5 and $6.25 \%)$ producing small but clear zones of inhibition at 100 and $50 \%$ extract. For $V$. cholerae Ogawa strain, the extracts used were SFE extract and methanol extracts, producing also small but clear inhibition zones (Fig. 1e and f).

\section{DISCUSSION}

Supercritical extraction with carbon dioxide is a feasible technique for the isolation of active substances from $N$. sativa. The supercritical extract is characterized by higher concentrations of the main extracted aromatic compounds thymoquinone. The SFE extract showed stronger antimicrobial activities against the tested bacteria $S$. aureus strains. The SFE extract had a very strong antimicrobial activity. This confirms the study of Agarwal et al. ${ }^{[2]}$ who reported that the oil inhibited one strain of $S$. aureus even up to 1:100 dilutions, the least concentration tested. However, the zones of inhibition observed in our study were larger which may be due to difference of the strains tested. The oil was found to be more effective on Gram positive than Gram negative bacteria, which is in conformity of a number of earlier studies compounds derived from plants often show considerable activity against Gram positive bacteria but not against Gram negative species. Gram-negative bacteria have an effective permeability barrier, comprised of the outer membrane, which restricts the penetration of amphipathic compounds and multidrug resistance pumps that extrude toxins across this barrier. It is possible that the apparent ineffectiveness of plant antimicrobials is largely due to the permeability barrier. Results of the study indicate that black seed oil possesses significant antimicrobial activity against Methicillin resistant Staphylococcus aureus (MRSA) and experiments evaluating the antibacteriall properties of black seed oil in suitable foods are underway in our laboratory. Super-Critical Fluids (SCFs) are increasingly replacing organic solvents, e.g., n-hexane, dichloromethane, chloroform and so on, that is conventionally used in industrial extraction, purification and recrystallization operations because of regulatory and environmental pressures on hydrocarbon and ozone-depleting emissions. In natural product extraction and isolation, Supercritical Fluid Extraction (SFE), especially that employing SFE, has become the method of choice. Sophisticated modern technologies allow precise regulation of changes in temperature and pressure and thus manipulation of solvating property of the SCF, which helps the extraction of natural products of a wide range of polarities. According to these advantages of supercritical fluid extraction we recommended to use as clean tool for essential oil extraction.

\section{REFERENCES}

1. Abduganiew, B.E., U.A. Abdullaev, K.N. Aripov, K.H.C. Baser and T. Oezek, 1997. Composition of the essential oil of Bunium persicum (Boiss.) B. Fedtsch. from Tajikistan. J. Essent. Oil Res., 9: 597-598. http://www.fao.org/agris/search/display.do?f=./199 8/v2407/US1997060315.xml;US1997060315

2. Agarwal, R., M.D. Kharya and R. Shrivastava, 1979. Antimicrobial and anthelmintic activities of the essential oil of Nigella sativa Linn. Indian J. Exp. Biol., 17: 1264-1265. http://www.ncbi.nlm.nih.gov/pubmed/549848

3. Agarwal, S.G., S. Ghosh, P.S. Jamwal, A.K. Raina and R.K. Thappa, 1991. Comparative studies on the major volatiles of kalazira (Bunium persicum seed) of wild and cultivated sources. Food Chem., 41: 129-134.

http://cat.inist.fr/?aModele $=$ afficheN\&cpsidt $=1978$ 8039

4. Ambroud, A., E. Reverchon and F. Senatore, 1994. Isolation of peppermint oil using supercritical $\mathrm{CO}_{2}$ extraction. Flavour Fragrance J., 9: 19-23. DOI: 10.1002/ffj.2730090105

5. Assis, L.M. and F.M. Lancas, 1999. Highresolution gas chromatography and high-resolution gas chromatography/mass spectrometry study of the volatile fraction obtained from high-inertinite Brazilian coal by supercritical fluid extraction. J. Microcolumn Separat., 11: 501-512. http://www3.interscience.wiley.com/journal/63001 207/abstract

6. Barnabas, I.J., J.R. Dean and S.P. Owen, 1994. Supercritical fluid extraction of analytes from environmental samples: A review. Analyst, 119: 2381-2394. http://cat.inist.fr/?aModele $=$ afficheN\&cpsidt $=3322$ 454 
7. Doraiswamy, L.K., S.R.S. Ferreira, M.A.A. Meireles, Z.L. Nikolov and A.J. Petenate, 1999. Supercritical fluid extraction of black pepper (Piper nigrun L.) essential oil. J. Supercrit. Fluids, 14: 235-245. DOI: 10.1016/S0896-8446(98)00092-8

8. Eikani, M.H., I. Goodarznia and M. Mirza, 1999. Supercritical carbon dioxide extraction of cumin seeds (Cuminum cyminum L.) Flavour Fragr. J., 14: $\quad 29-31$. DOI: $10.1002 /($ SICI $1099-$ 1026(199901/02)

9. Fekete, J., A. Kery, E. Lemberkovics, M. Oszagyan, J. Sawinsky and B. Simandi, 1996. Supercritical fluid extraction of volatile compounds from lavender and thyme. Flavour Frag. J., 11: 157-165. DOI: 10.1002/(SICI)1099-1026(199605)

10. Mashhadian, N.V. and H. Rakhshandeh, 2005. Antibacterial and antifungal effects of Nigella sativa extracts against $S$. aureus, Ps. aeruginosa and C. albicans. Pak. J. Med. Sci., 21: 47-52. http://www.pakmedinet.com/6871

11. Mincioned, B., M. Poiana and V. Sicari, 1998. Supercritical carbon dioxide $\left(\mathrm{SC}-\mathrm{CO}_{2}\right)$ extraction of grapefruit flavedo. Flavour Fragr. J., 13: 125-130. http://cat.inist.fr/?aModele=afficheN\&cpsidt=2236555
12. Modey, W.K., D.A. Mulholland and M.W. Raynor, 1996. Review: Analytical supercritical fluid extraction of natural products. Phytochem. Anal., 7: 1-15. http://www3.interscience.wiley.com/journal/22854/ abstract

13. National Committee for Clinical Laboratory Standards (NCCLS). 2002. Performance standards for antimicrobial susceptibility testing. NCCLS approved standard M100-S12. National Committee for Clinical Laboratory Standards, Wayne, Pa.

14. PourmortazavI, S.M., F. Sefidkon and S.G. Hosseini, 2003. Supercritical carbon dioxide extraction of essential oils from Perovskia atriplicifolia Benth. J. Agric. Food Chem., 51: 5414-5419. DOI: $10.1021 / \mathrm{jf0341619}$

15. Ferdous, A.J. and S.N. Islam, 1992. In vitro antibacterial activity of the volatile oil of Nigella Sativa seeds against multiple drug- resistant isolates of Shigella spp. and isolates of Vibrio cholerae and E. coli. Phytother. Res., 6: 137-140. http://cat.inist.fr/?aModele $=$ afficheN\&cpsidt $=5254$ 460 